\begin{tabular}{|c|l|}
\hline Title & Fractal encoding in a chaotic neural network \\
\hline Author(s) & Ryeu, J. K.; A ihara, K.; Tsuda, I. \\
\hline Citation & $\begin{array}{l}\text { Physical Review E, 64(4), 046202-1-046202-6 } \\
\text { https://doi.org/10.1103/PhysRevE.64.046202 }\end{array}$ \\
\hline Issue Date & 2001-09 \\
\hline Doc URL & http://hdl.handle.net/2115/8487 \\
\hline Rights & Copyright (c) 2001 The A merican Physical Society \\
\hline Type & article \\
\hline File Information & e046202.pdf \\
\hline
\end{tabular}

Instructions for use 


\title{
Fractal encoding in a chaotic neural network
}

\author{
J. K. Ryeu, ${ }^{1, *}$ K. Aihara, ${ }^{2, \dagger}$ and I. Tsuda ${ }^{3, *}$ \\ ${ }^{1}$ Department of Electronic Engineering, Dongyang University, Youngju, Korea \\ ${ }^{2}$ Graduate School of Frontier Science, The University of Tokyo and CREST, JST, Tokyo, Japan \\ ${ }^{3}$ Department of Mathematics, Graduate School of Science, Hokkaido University, Sapporo, Japan
}

(Received 8 March 2001; published 19 September 2001)

\begin{abstract}
We analyze a model of a chaotic neural network consisting of three neurons, namely a chaotically forcing neuron and two neurons comprizing a stable response system with a contraction mapping property, for digital encoding with chaotic dynamics. We show that dynamics of the chaotically forcing neuron is embedded in the form of a code sequence on a fractal attractor of the two-neuron response system. We consider the relation between the state transition of the chaotically forcing neuron and the hierarchical fractal structure on the attractor in the state space of the contracting system. We also report hardware implementation of the presented model with an analog electronic circuit to investigate the fractal attractor of the chaotic neural network as a realistic system.
\end{abstract}

DOI: 10.1103/PhysRevE.64.046202

PACS number(s): 05.45.Df, 05.45.Gg, 05.45.Vx, 87.18.Sn

\section{INTRODUCTION}

There are many studies on the relations between chaos and fractals [1-6]. Rössler et al. [7] reported some models representing chaos-driven contraction mapping. Their paper describes a hierarchy of models exhibiting fractal attractors including strange nonchaotic attractors found by Grebogi et al. and singular-continuous nowhere-differentiable (SCND) attractors, too. Tsuda [8], and Tsuda and Yamaguchi [9] have also found the SCND attractor in a neural system consisting of chaotic neuron models proposed by Aihara et al. [10]. Their study shows that a Cantor-like attractor is observed on a cross section with respect to an output of the forcing neuron in the chaotic neural network. On the other hand, a lot of research on the use of chaos for nonlinear digital communications, especially for the encoding of digital information, has been reported [11-14]. Based on this research, we can expect that if a dynamical system has welldefined symbolic dynamics, the encoding of digital information is accomplished using the principle of controlling chaos $[15,16]$. Furthermore, Tsuda [8,17], and Tsuda and Kuroda [18] showed that the transition of the states of a forcing chaotic neuron can be hierarchically labeled on the fractal attractor of a chaotically forced response system.

The purpose of the present paper is to further develop fractal symbolic encoding in a chaotically forced contraction system that exhibits fractal attractors, by modifying the Tsuda-Yamaguchi model $[8,9,17]$. To clarify the encoding property, we introduce hard-limit functions, or Heaviside functions as transfer functions from the forcing neuron to the response system, thereby the system is converted to an IFS (Iterated Function System)-like model that is composed of not affine but rather nonlinear transformations. According to Barnsley [19], if the IFS is totally disconnected and if the points on the attractor are distributed sparsely, it is possible

\footnotetext{
*Email address: jkryeu@phenix.dyu.ac.kr

†Email address: aihara@sat.t.u-tokyo.ac.jp

‡Email address: tsuda@math.sci.hokudai.ac.jp
}

to improve memories with a very high storage capacity and robustness against noise. Although the transfomation of the proposed system is nonlinear rather than affine, and it may not be completely invertible, a kind of coding of information may also be possible $[9,17]$. We also implement the hardware to clarify the dynamics of the system in a real electronic circuit. The circuit consists of electronic chaotic neuron models [20], connection weights, and comparators for the hard-limit functions.

In Sec. II, we propose a model for the present investigation, containing the model equations. In Sec. III, we clarify the relation between the structure of the code and the hierarchical structure of a fractal attractor, and we also discuss the Lyapunov dimension as a dynamical feature of the network. Section IV contains the hardware implementation of the system and the experimental results. Section V is devoted to the discussion on the meaning of fractal encoding on the attractor and the possibility of its application to information processing.

\section{THE MODEL NETWORK}

We consider the nonlinear dynamics of a type of chaotic neural network [10], which consists of a chaotically forcing neuron and two almost linear neurons. The latter stable neurons are forced by the former chaotic neuron through transfer functions as shown in Fig. 1. In the present model, we use

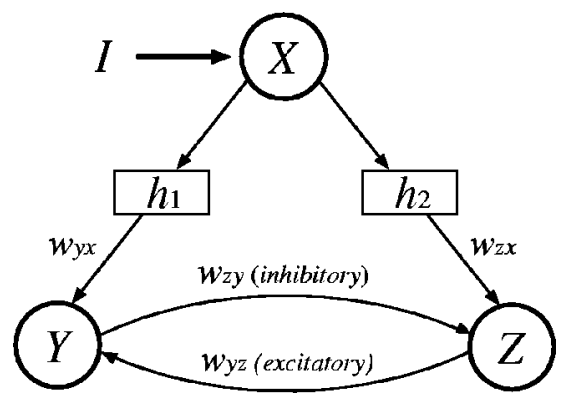

FIG. 1. Network configuration of a chaotically forced contracting system. 
two hard-limit functions, or Heaviside functions as transfer functions from the chaotic neuron $X$ to the static neurons $Y$ and $Z$ to encode the dynamics of the chaotic neuron. The network equations are as follows $[8-10]$ :

$$
\begin{aligned}
x_{n+1}=f_{1}\left(-\alpha_{1} \sum_{r=0}^{n} k_{1}^{r} x_{n-r}+I_{0}\right) \\
y_{n+1}=f_{2}\left(-\alpha_{2} \sum_{r=0}^{n} k_{2}^{r} y_{n-r}+w_{y z} \sum_{r=0}^{n} k_{2}^{r} z_{n-r}\right. \\
\left.+w_{y x} \sum_{r=0}^{n} k_{2}^{r} h_{1}\left(x_{n-r}\right)\right) \\
z_{n+1}=f_{3}\left(-\alpha_{3} \sum_{r=0}^{n} k_{3}^{r} z_{n-r}+w_{z y} \sum_{r=0}^{n} k_{3}^{r} y_{n-r}\right. \\
\left.+w_{z x} \sum_{r=0}^{n} k_{3}^{r} h_{2}\left(x_{n-r}\right)\right),
\end{aligned}
$$

where $\alpha_{i}$ is a positive parameter, $I_{0}$ is the strength of the external input to the neuron $X, k_{i}$ is a decay parameter with $0<k_{i}<1(i=1,2,3), w_{v u}$ is the connection weight from neuron $u$ to $v$ with $w_{z y}<0$, and $w_{y z}, w_{y x}, w_{z x}>0$, and the function $f_{i}(x) \quad(i=1,2,3)$ is the following sigmoidal function:

$$
f_{i}(x)=\frac{1}{1+e^{-x / \epsilon_{i}}}, \quad(i=1,2,3),
$$

where $\epsilon_{i}$ is a steepness parameter.

The hard-limit transfer functions $h_{i}(x)(i=1,2)$ are defined as follows:

$$
\begin{aligned}
& h_{1}(x)= \begin{cases}0 & (x<a) \\
1 & (x \geqslant a),\end{cases} \\
& h_{2}(x)= \begin{cases}0 & (x<b) \\
1 & (x \geqslant b),\end{cases}
\end{aligned}
$$

where $a$ and $b$ are the threshold parameters assumed to be $a<b$ in this paper. The functions $h_{i}(x)(i=1,2)$ represent wave-shaping effect of axons producing all-or-none behaviors of propagating action potentials [10].

These equations can be reduced by variable transformations [10] with defining new variables $X_{n+1}, Y_{n+1}$, and $Z_{n+1}$ as follows:

$$
\begin{gathered}
X_{n+1}=-\alpha_{1} \sum_{r=0}^{n} k_{1}^{r} x_{n-r}+I_{0}, \\
Y_{n+1}=-\alpha_{2} \sum_{r=0}^{n} k_{2}^{r} y_{n-r}+w_{y z} \sum_{r=0}^{n} k_{2}^{r} z_{n-r} \\
+w_{y x} \sum_{r=0}^{n} k_{2}^{r} h_{1}\left(x_{n-r}\right),
\end{gathered}
$$

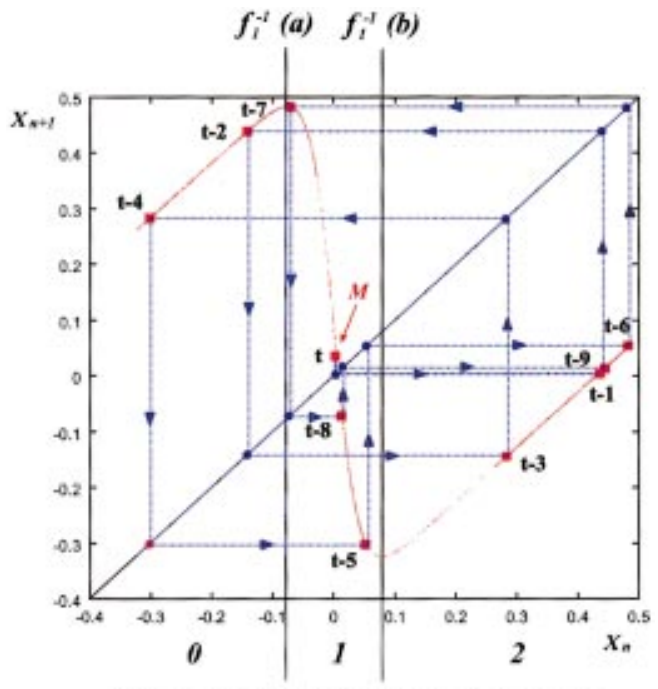

Code sequence of $M: 1202012112 \ldots$

FIG. 2. (Color) Definition of a code sequence based on the chaotic neuron map [10].

$$
\begin{aligned}
Z_{n+1}= & -\alpha_{3} \sum_{r=0}^{n} k_{3}^{r} z_{n-r}+w_{z y} \sum_{r=0}^{n} k_{3}^{r} y_{n-r} \\
& +w_{z x} \sum_{r=0}^{n} k_{3}^{r} h_{2}\left(x_{n-r}\right) .
\end{aligned}
$$

Then we can represent the dynamics of the network as follows:

$$
\begin{gathered}
X_{n+1}=k_{1} X_{n}-\alpha_{1} f_{1}\left(X_{n}\right)+I, \\
Y_{n+1}=k_{2} Y_{n}-\alpha_{2} f_{2}\left(Y_{n}\right)+w_{y z} f_{3}\left(Z_{n}\right)+w_{y x} h_{1}\left[f_{1}\left(X_{n}\right)\right],
\end{gathered}
$$

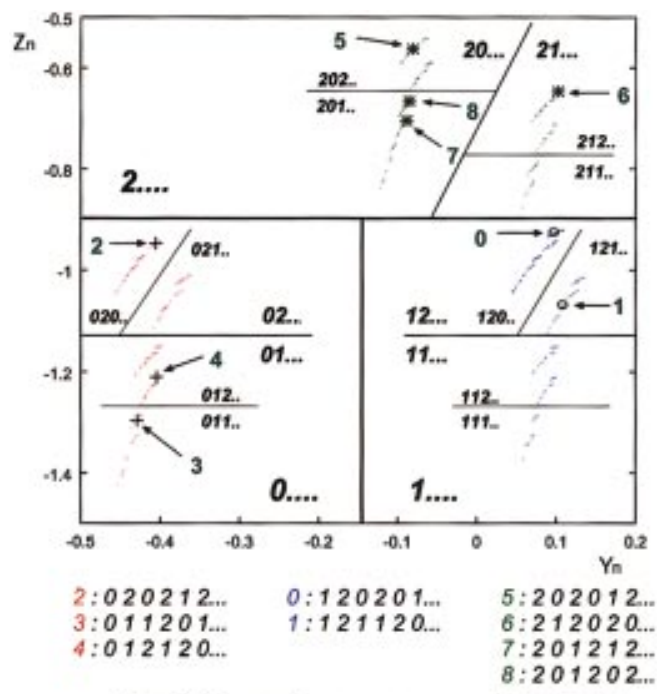

Forbidden subsequences : 00, 10, 22

FIG. 3. (Color) Hierarchy of codes on the fractal attractor for $I$ $=0.58$. 


$$
Z_{n+1}=k_{3} Z_{n}-\alpha_{3} f_{3}\left(Z_{n}\right)+w_{z y} f_{2}\left(Y_{n}\right)+w_{z x} h_{2}\left[f_{1}\left(X_{n}\right)\right]
$$

where $I=I_{0}\left(1-k_{1}\right)$, which is used to control chaotic dynamics of the neuron $X$.

By choosing $a$ and $b$ in Eqs. (5) and (6) in accordance with the two critical points of the chaotic neuron map [10] of $X$ as shown in Fig. 2, the information from a value of $X$ can be transferred and used to stimulate $Y$ and $Z$ via $\left(h_{1}, h_{2}\right)$ $=\{(0,0),(1,0),(1,1)\}$, according to the condition that the value $X$ is less than $a$, between $a$ and $b$, or more than $b$. The three subintervals divided by the two critical points in the one-dimensional map are labeled in Fig. 2 as 0,1 , and 2. We use the parameter values $k_{1}=0.98, k_{2}=k_{3}=0.8, \epsilon_{1}=0.02$, $\epsilon_{2}=\epsilon_{3}=0.5, \quad \alpha_{1}=\alpha_{2}=\alpha_{3}=1, \quad I=0.58, \quad w_{y x}=w_{z x}=w_{y z}$ $=0.5$, and $w_{z y}=-0.5$ in the simulation.

\section{FRACTAL ENCODING}

\section{A. Code sequence and fractal attractor}

We next consider the relation between the structure of a code and that of an attractor. We divide the region of the state in the chaotic neuron map into three subintervals and label the symbols 0, 1, and 2 for each one as shown in Fig. 2 [17]. We then express the dynamical series $\left\{X_{n}\right\}$ as a code sequence consisting of the symbols 0,1 , and 2 . For example, the point $M$ on the chaotic map in Fig. 2, which corresponds to the point 0 on the attractor shown in Fig. 3, can be labeled as $1202012112 \ldots$. Figures $3-5$ show the hierarchical relation between the codes generated by the symbolic dynamics of the forcing chaotic neuron and the structure of the attractor, for various values of the external input $I$. As these figures show, the attractors have self-similar fractal structures where

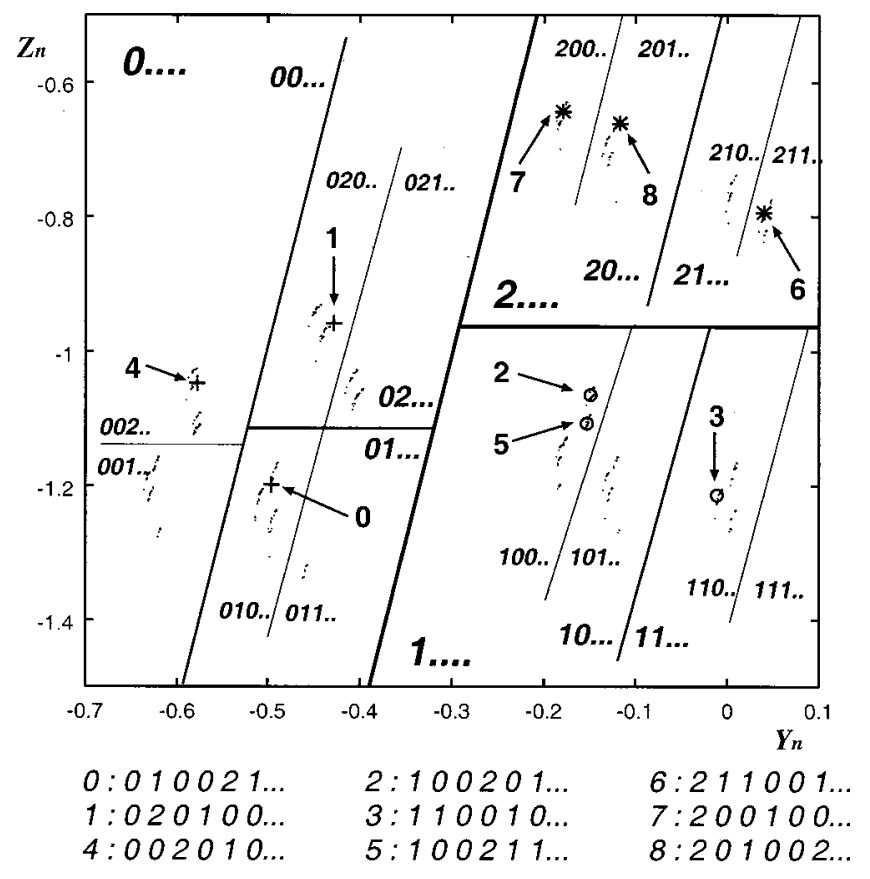

Forbidden subsequences : 12, 22

FIG. 4. Hierarchy of codes on the fractal attractor for $I=0.29$.

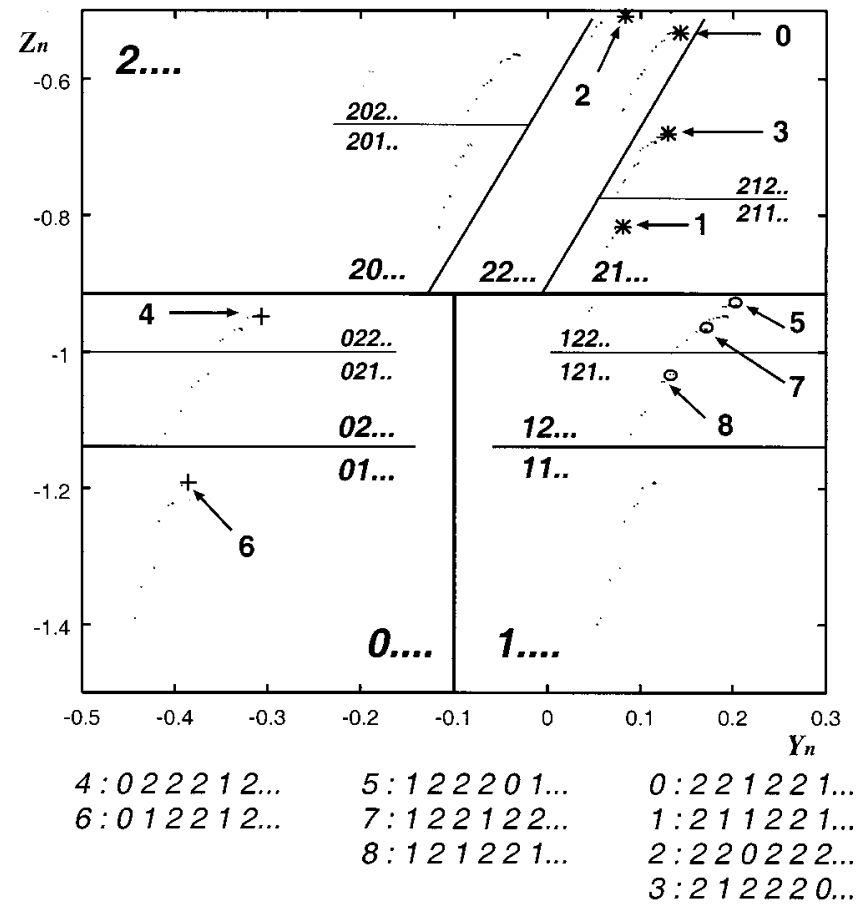

Forbidden subsequences : 00, 10

FIG. 5. Hierarchy of codes on the fractal attractor for $I=0.78$.

the hierarchy of the structure of the attractor corresponds to the hierarchy of the symbolic codes generated by the forcing chaotic neuron.

There are some types of transitions among symbols, which cannot be generated by the dynamics of the chaotic neuron $X$, depending on values of the external input $I$ as well as the internal parameters of the chaotic neuron, $\epsilon_{1}, \alpha_{1}$, and $k_{1}$. For example, at $\epsilon_{1}=0.02, \alpha_{1}=1, k_{1}=0.98$, and

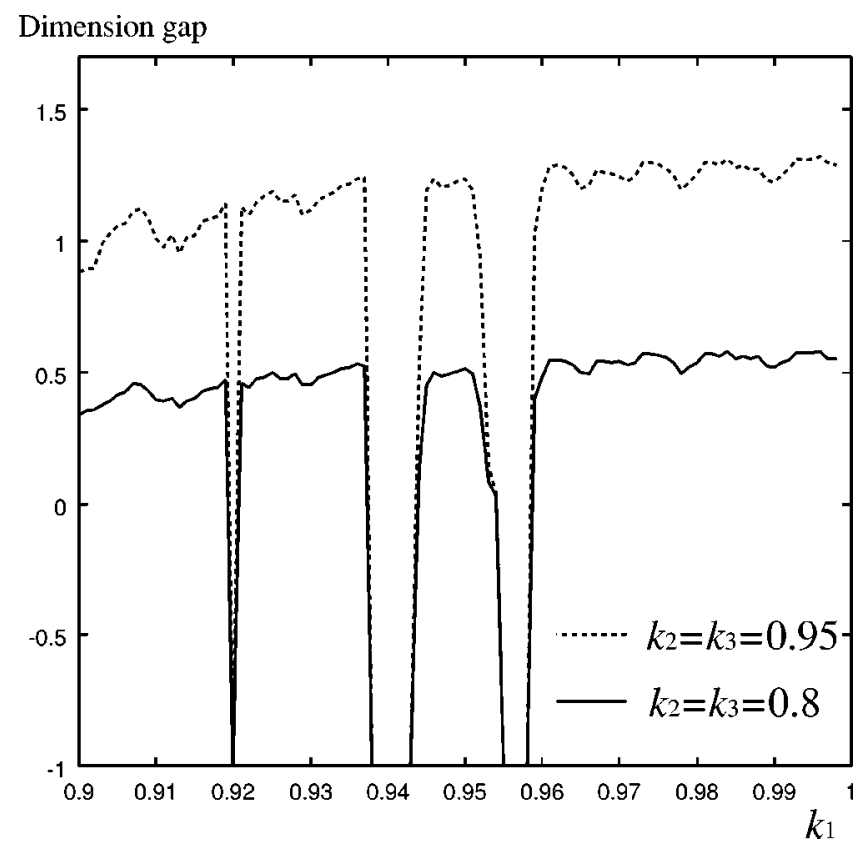

FIG. 6. Dimension gap versus $k_{1}$. 


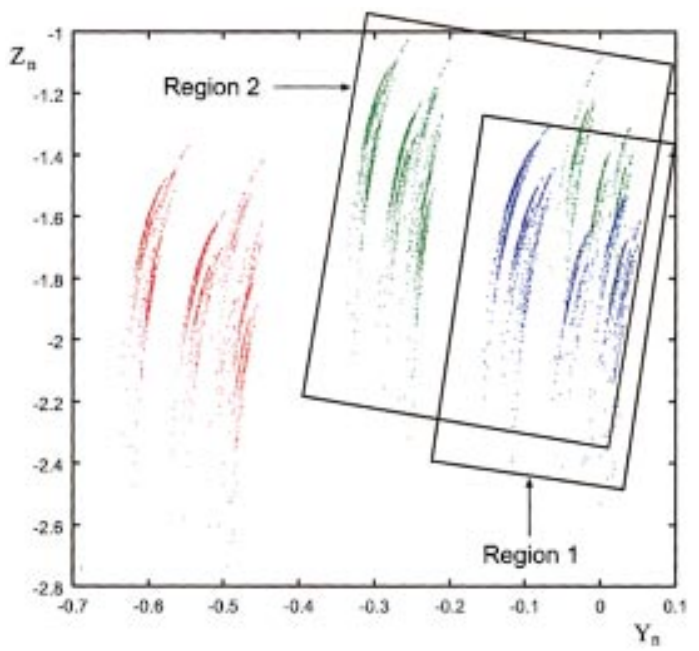

FIG. 7. (Color) An overlapped fractal attractor for $k_{1}=0.98$, $k_{2}=k_{3}=0.95$.

$I=0.58$, the transitions $0 \rightarrow 0,0 \rightarrow 1$, and $2 \rightarrow 2$ are forbidden, therefore no subsequences such as 00,10 , and 22 appear in the code sequence.

The correspondence of a symbolic code to an invariant set has been studied, using the technique of symbolic dynamics [21]. Hence it may be possible for a real number to have one-to-one correspondence to each point on a fractal attractor in the proposed system. However, since the response system consists of contractive maps, that is, all the Lyapunov exponents of the inverse mapping must be positive, the length of a code sequence to be retrieved(decoded) from an attractor may not be infinite due to instability and inevitable noise.

\section{B. Dynamic feature of the network}

To consider the stability and disconnectivity of the system, we next calculate the dimension gap [8,9,22], defined as the difference between the Hausdorff dimension and the topological dimension, as a measure of the dispersion of an attractor. Since the Hausdorff dimension is not computable, the Lyapunov dimension can alternatively be used for practical demands. The Lyapunov dimension of an attractor $A$ is calculated using the following formula:

$$
\operatorname{dim}_{\lambda} A=m+\frac{\sum_{i=1}^{m} \lambda_{i}}{\left|\lambda_{m+1}\right|},
$$

where $\lambda_{1} \geqslant \lambda_{2} \geqslant \cdots \geqslant \lambda_{n}$ are the Lyapunov exponents and $m$ is determined as

$$
\sum_{i=1}^{m} \lambda_{i} \geqslant 0, \quad \sum_{i=1}^{m+1} \lambda_{i}<0 .
$$

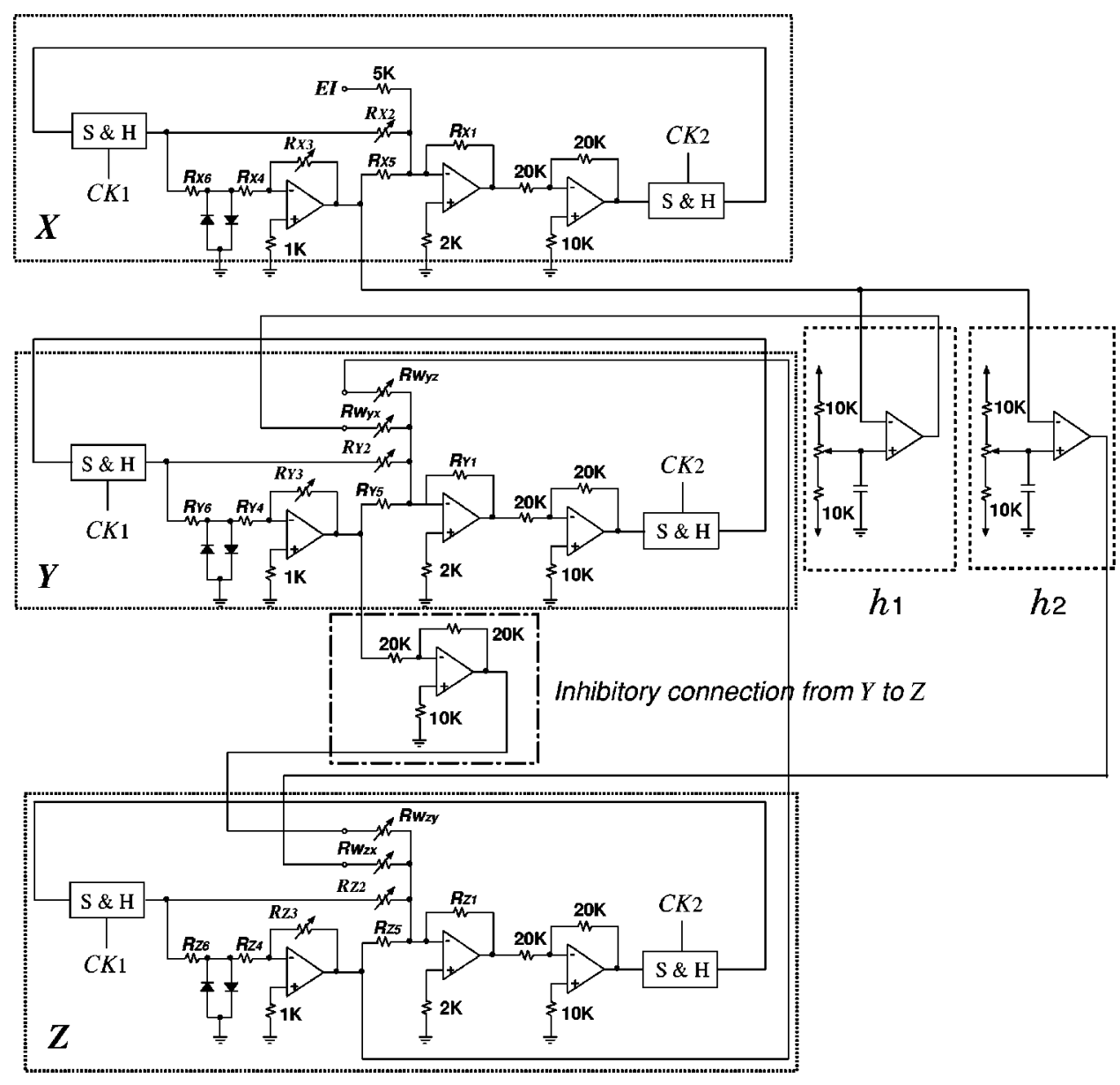

FIG. 8. Circuit diagram of the network. 


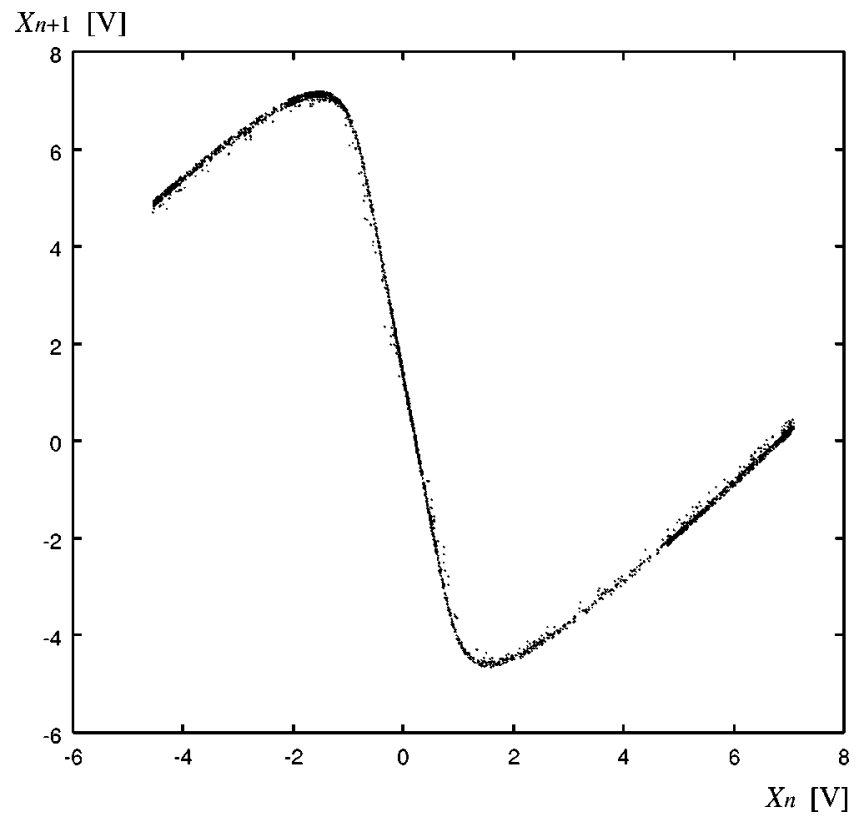

FIG. 9. A return map of a chaotic neuron implemented with an analog electronic circuit.

In the present network, the topological dimension is unity because only the forcing system is chaotic, and the response system is contractive. Figure 6 shows the characteristics of the relation between the dimension gap and $k_{1}$. According to Rössler et al. [7,22], the dimension gap should be greater than unity for a singular-continuous nowhere-differentiable attractor. Figure 6 implies that the values of $k_{2}$ and $k_{3}$ must be chosen to be large enough to satisfy this condition. Tsuda and Yamaguchi [9] also discussed the idea that large values of the dimension gap may be advantageous for information

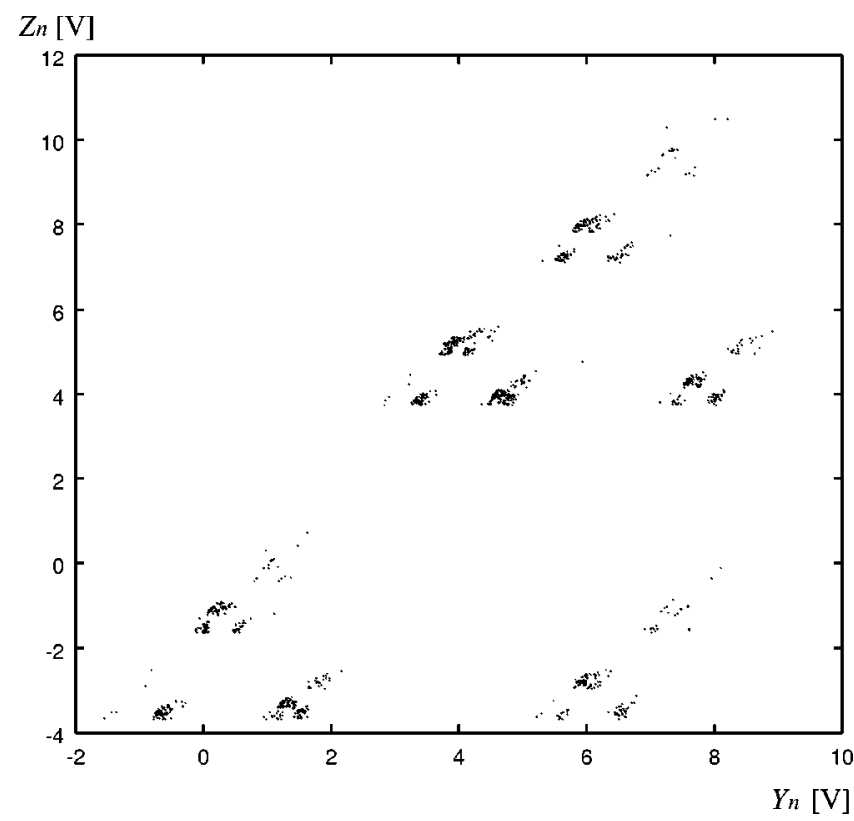

FIG. 10. Fractal-like structure observed on the $Y_{n}-Z_{n}$ space in an analog electronic circuit of a chaotically forced contracting system. processing on the fractal attractor under noisy conditions, namely the stability of encoding.

On the other hand, if the value of the dimension gap is too large, the disconnectivity, or the non-overlapping property of the attractor is destroyed, as demonstrated in Fig. 7. Thus, there must be some trade off between the stability of coding and the total disconnectivity in determining the contraction rates $k_{2}$ and $k_{3}$.

\section{HARDWARE IMPLEMENTATION AND EXPERIMENTAL RESULTS}

\section{A. System configuration}

The circuit diagram of the system is shown in Fig. 8. The system consists of three neurons [20], one chaotic, and the other two almost linear, two comparators for the hard-limit functions, and some resistors for connection weights. The major parameters of a neuron, the attenuation constant $k_{i}$ and the steepness $\epsilon_{i}$ of the nonlinear function can be adjusted as

$$
\begin{gathered}
k_{i}=\frac{R_{A 1}}{R_{A 2}}, \\
\epsilon_{i} \simeq\left[4\left\{\frac{R_{A 1}}{R_{A 2}}-\frac{R_{A 1} R_{A 3}}{\left(R_{A 4}+R_{A 6}\right) R_{A 5}}\right\}\right]^{-1},
\end{gathered}
$$

where $(i, A)=\{(1, X),(2, Y),(3, Z)\}$.

The contraction rates, the slopes of the nonlinear functions, and the connection weights between neurons are chosen according to the following conditions: $0.9<k_{1}<1.0$, $0.8 \leqslant k_{i}<1.0 \quad(i=2,3), \quad \epsilon_{1}<0.05, \quad \epsilon_{i} \geqslant 0.5 \quad(i=2,3), \quad 0$ $<w_{y x}, w_{z x}, w_{y z}<1$, and $-1<w_{z y}<0$.

\section{B. Experimental results}

Figure 9 shows a return map for the voltage of the output $X$ in the analog circuit. The data were obtained by $A / D$ conversion with $10 \mathrm{kHz}$ of sampling frequency and 14 bit of quantizing resolution. The horizontal and vertical axes represent $X_{n}$ and $X_{n+1}$, respectively.

Figure 10 shows the fractal-like structure on the observed $Y_{n}-Z_{n}$ space. The parameter values $k_{i}$ and $\epsilon_{i}$ were set as follows.

The chaotic driving neuron: $k_{1}=0.98, \epsilon_{1}=0.02$; the quasilinear response neurons: $k_{2}=k_{3}=0.8, \epsilon_{2}=\epsilon_{3}=0.5$. The connection weights are $w_{y x}=w_{z x}=0.5, w_{y z}=0.5$, and $w_{z y}$ $=-0.5$ in the experiment.

\section{DISCUSSION AND CONCLUSION}

In the present study, we proposed a model of chaotic neural network for fractal encoding and analyzed the nonlinear dynamics, by modifying the model proposed by Tsuda and Yamaguchi $[8,9]$. We also implemented the proposed network as a hardware system with analog discrete devices to investigate whether or not the fractal encoding is actually realized. The system works in bipolar mode and can not be free from the noise inherent in discrete analog circuits, thus 
the attractor in the experiment is slightly different in shape from the simulated attractor. In spite of this deformation, the fractal attractor structure with three regions 0,1 , and 2 is clearly demonstrated. We also observed by this hardware the robustness of the fractal attractor for noise to a certain degree.

In future study, the invertibility of the mapping for decoding must be examined. The relation between the distance in the sequences of codes and the spatial distance on the attractor should be also investigated in detail. Another problem, which should be interesting from the viewpoint of engineering, is to apply the concept of encoding on the fractal attrac- tor to practical information processing, e.g., coding and compressing digital information. In this respect, the construction of electronic integrate circuits with parameter-controllable chaotic neuron chips will be an extremely important subject for study.

\section{ACKNOWLEDGMENTS}

The authors thank A. Yamaguchi, Y. Horio, N. Ichinose, H. Shiraishi, K. Fukuda, and M. Okubo for their valuable discussions and comments.
[1] M. Hata and M. Yamaguti, Jpn. J. Appl. Math. 1, 186 (1984).

[2] M. Yamaguti, J. Fac. Sci., Univ. Tokyo 36, 187 (1989).

[3] J. Moser, J. Diff. Eqns. 5, 411 (1969).

[4] C. Grebogi, E. Ott, S. Pelikan, and J.A. Yorke, Physica D 13, 261 (1984).

[5] K. Kaneko, Collapse of Tori and Genesis of Chaos in Dissipative Systems (World Scientific, Singapore, 1986), p. 118.

[6] J.L. Kaplan and J.A. Yorke, Lect. Notes Math. 730, 204 (1979).

[7] O.E. Rössler, J.L. Hudson, C. Knudsen, and I. Tsuda, J. Intell. Syst. 10, 15 (1995).

[8] I. Tsuda, Int. J. Neural Syst. 7, 451 (1996).

[9] I. Tsuda and A. Yamaguchi, Neural Networks 11, 927 (1998).

[10] K. Aihara, T. Takabe, and M. Toyoda, Phys. Lett. A 144, 333 (1990).

[11] S. Hayes, C. Grebogi, and E. Ott, Phys. Rev. Lett. 70, 3031 (1993).

[12] S. Hayes, C. Grebogi, E. Ott, and A. Mark, Phys. Rev. Lett.
73, 1781 (1994).

[13] E. Bolt and Y.C. Lai, Phys. Rev. E 58, 1724 (1998).

[14] Y.C. Lai, Int. J. Bifurcation Chaos Appl. Sci. Eng. 10, 787 (2000).

[15] E. Ott, C. Grebogi, and J.A. Yorke, Phys. Rev. Lett. 64, 1196 (1990).

[16] L.M. Pecora and T.L. Carroll, Phys. Rev. Lett. 64, 821 (1990).

[17] I. Tsuda, Behav. Brain Sci. 24, 575 (2001).

[18] I. Tsuda and S. Kuroda, Jpn. J. Indus. Appl. Math. 18, 249 (2001).

[19] M. Barnsley, Fractals Everywhere (Academic Press, New York, 1988), pp. 118-134.

[20] K. Shimizu, K. Aihara, and M. Kotani, Electron. Commun. Jpn. Scripta Technica 3 74, 51 (1991).

[21] B.L. Hao and W.M. Zheng, Directions in Chaos 7, 103 (1998).

[22] O. E. Rössler, R. Wais, and R. Rössler, in Proceedings of the Second International Conference on Fuzzy Logic and Neural Networks, Iizuka, Japan, 1992, p. 909. 